\title{
Ptosis mamaria y su resolución quirúrgica
}

\author{
Breast ptosis and surgical resolution
}

\author{
Lucas Bellini Ditta
}

REVISTA ARGENTINA DE CIRUGÍA PLÁSTICA 2018;24(2):95-104. DOI/10.32825/RACP/201802/0095-0104

\section{ÍNDICE}

1. Resumen/Summary . .3

2. Objetivo y palabras clave ...............................................

3. Introducción..................................................................

4. Clasificación .....................................................................

5. Embriología de la mama .................................................6

6. Anatomía de la mama ......................................................

7. Posicionamiento topográfico.......................................

8. Pexia mamaria en mama hipertrófica .......................9

9. Técnicas a elegir según ptosis con hipertrofia .... 14

10. Pexia mamaria en glándula hipotrófica ................ 16

11. Conclusión.............................................................. 19

12. Bibliografía .................................................................... 19

\section{RESUMEN}

La ptosis mamaria es una anomalía de la mama relativamente frecuente, que se debe resolver con reconstrucción quirúrgica. Los objetivos de la reconstrucción quirúrgica de esta anomalía son dos: fundamentalmente corregir la posición inestética de la mama, y en segundo plano darle consorte al posible déficit funcional que pueda presentar

En general, los déficits funcionales son tratados inmediatamente, mientras que la reparación cosmética puede dividirse en dos momentos vitales del paciente; el final de la pubertad en aquellos casos vinculados al crecimiento excesivo de la glándula y consecuente pérdida de posicionamiento adecuado del CAP para alcanzar la simetría máxima posible después de la maduración completa de la mama con la reducción pertinente de la glándula, o bien según la solicitud del paciente adulto que busca recuperar la imagen estética personal perdi-

1. Posgrado en Cirugía Plástica y Reconstructiva. Rotación en Cirugía Estética. Universidad del Salvador.

$\triangle$ Correspondencia: revista@sacper.com.ar

Los autores no declaran conflictos de intereses

Recibido: 09/09/2018 / Aceptado: 02/10/2018 da con el curso de los años a partir de la atrofia glandular o elongación excesiva de los tejidos.

\section{SUMMARY}

Mammary ptosis is a relatively frequent breast anomaly, which must be resolved with surgical reconstruction. The objectives of the surgical reconstruction of this anomaly are two: fundamentally correct the unstatic position of the breast, and in the background give consolation to the possible functional deficit that may present.

In general, functional deficits are treated immediately, while cosmetic repair can be divided into two vital moments of the patient; the end of puberty in those cases related to the excessive growth of the gland and consequent loss of adequate positioning of the CAP to reach the maximum possible symmetry after the complete maturation of the breast with the relevant reduction of the gland, or according to the request of the adult patient who seek store cover the lost personal aesthetic image over the years from glandular atrophy or excessive tissue elongation.

\section{OBJETIVO}

Realizar una revisión anatómica de la estructura glandular, así; como de las principales técnicas quirúrgicas que pueden utilizarse para la resolución del inesteticismo ptósico glandular tanto de mujeres jóvenes como adultas.

Palabras clave: anatomía, reposicionamiento, inestética, hipertrofia glandular, atrofia glandular

\section{INTRODUCCIÓN}

La ptosis mamaria es una afección frecuente, dentro del desarrollo evolutivo que sufre la glándula mamaria y que conlleva una caída de la misma. En asociación con hipertrofia glandular, puede tornarse invalidante. Los pedidos de corrección son muy numerosos, siendo una importante actividad para muchos cirujanos plásticos. En la etiológica hay una pérdida de elasticidad en 


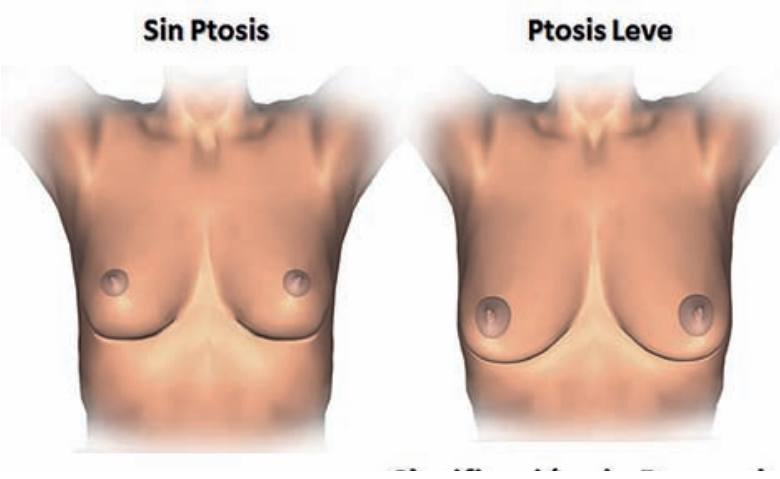

Figura 1. Clasificación de Regnault para la ptosis mamaria.

la dermis y de una pérdida de la masa glandular tras la lactancia, edad y/o pérdida de peso, o bien un excesivo tamaño y consecuente peso que llevan al descenso glandular. La intervención que se realiza para solucionar este problema es la que se conoce con el nombre de Mastopexia. La palabra Mastopexia deriva del griego mastos (mama) y pexia (fijación). Los riesgos de esta cirugía deben ser mínimos, así como las complicaciones, desde las simples correcciones cicatrízales de reposicionamiento en mamas elongadas (atróficas), como en las cicatrices con tensión tisular de mamas ptosadas por hipertrofia glandular. De aquí la importancia en los diferentes diseños quirúrgicos seleccionados en base a cada paciente y criterio del cirujano.

\section{CLASIFICACIÓN}

Regnault clasifica la ptosis mamaria en (Figura 1): Grado I.- Areolas a nivel del surco submamario.

Grado II.- Areolas por debajo del nivel del surco submamario.

Grado III.- Areolas muy por debajo del surco submamario y orientadas hacia abajo.

Cuando el complejo areola-pezón se encuentra en una posición adecuada, pero la distancia pezón-surco está aumentada, se conoce como pseudoptosis o ptosis falsa. En la pseudoptosis, la mama tendrá un aspecto caído, pero a expensas de que la glándula se encuentra herniada en el polo inferior, apareciendo el polo superior vacío. Se tratará de una forma distinta a la ptosis verdadera, por lo que hay que diferenciarlas $\left.{ }^{27}\right]$.

Puckett, en 1985, las clasifica en (Figura 2):

GRADO I: mama ptósica pero con la areola por encima del pliegue inframamario.

GRADO I $1 \frac{1}{2}$ :mama ptósica y areola que sobrepasa por debajo del pliegue.

GRADO II: mama ptósica y areola que desciende por debajo del pliegue inframamario.

GRADO III a: hay una ptosis severa, el complejo areola-pezón ocupa una posición por debajo del pliegue inframamario.
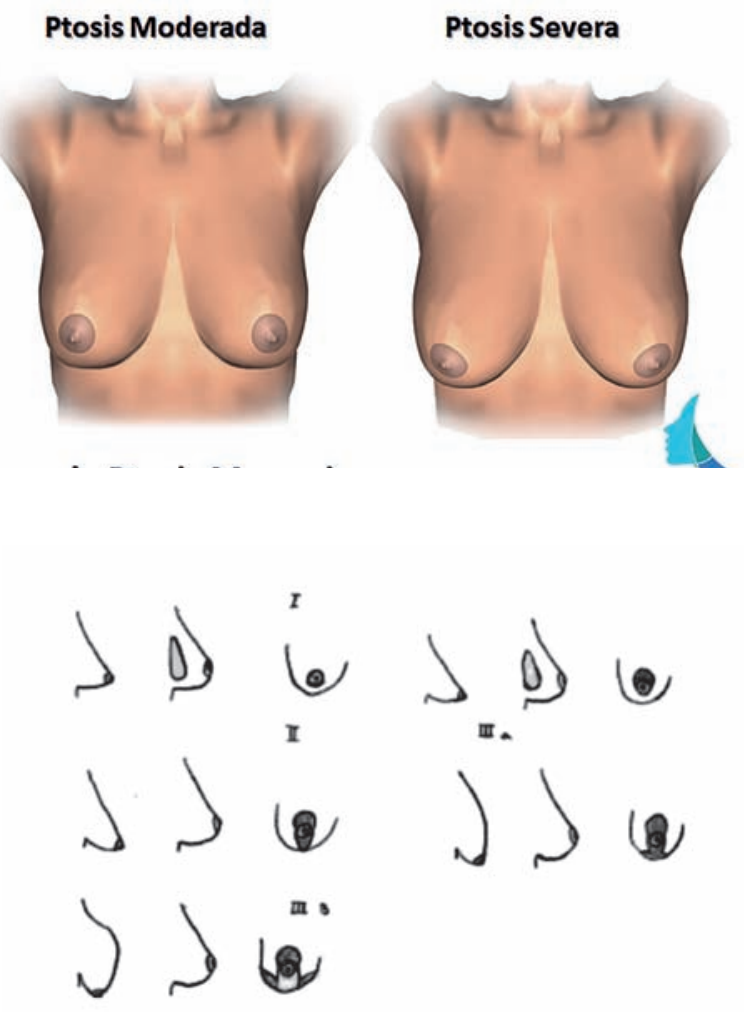

Figura 2. Clasificación de Pluckett para la ptosis mamaria.

GRADO IIIb: ptosis muy severa y complejo areola-pezón en posición inferior y posterior al pliegue inframamario $^{17}$.

\section{EMBRIOLOGÍA DE LA MAMA}

La mama comienza a formarse entre las semanas 5 y 7 del desarrollo fetal, como un engrosamiento bilateral del ectodermo, llamada línea mamaria, que involuciona un poco después de su formación. Sin embargo, una parte limitada permanece en la región torácica y constituye la base para desarrollo de la mama neonatal ${ }^{1}$. El engrosamiento de la línea mamaria restante forma el reborde mamario, seguido por la etapa en la cual el tejido epitelial se invagina en el mesénquima subyacente, a las 8 semanas de gestación. Posteriormente, el tejido epitelial sigue creciendo e invade más profundo en la pared mesenquimal torácica, y el tejido epitelial comienza a formar brotes, que se bifurcan en los alvéolos primitivos de la mama en el estadio de la bifurcación. En la semana 16, la etapa de la bifurcación ha producido aproximadamente 15-25 brotes. La diferenciación de los tejidos ocurre cuando maduran las glándulas sudoríparas y las glándulas sebáceas. Cuando el feto alcanza el tercer trimestre de gestación, comienza la etapa de canalización del desarrollo, que es la primera etapa de desarrollo de la mama provocada por influencias hormonales. Las hormonas placentarias sexuales median la canalización de los brotes de epitelio antes formados, para completar la formación 

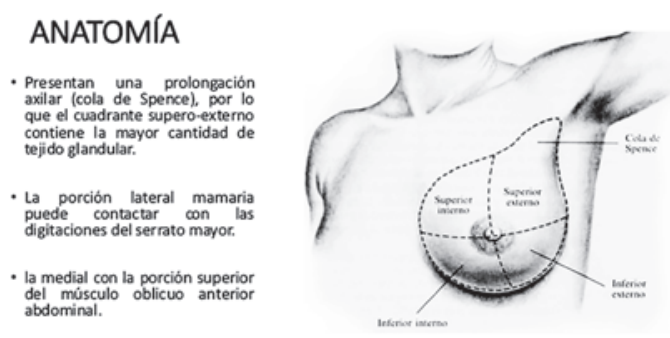

Figura 3. Anatomía.

de los conductos primitivos mamarios. La etapa final del desarrollo fetal de la mama comprende la diferenciación del parénquima mamario en lóbulos, con el desarrollo del complejo areola-pezón ${ }^{2}$. Esta etapa comienza en la semana 32 y continúa hasta el nacimiento. La canalización y la bifurcación del tejido mamario siguen en la fase temprana de la niñez. Hay fuerte evidencia del papel del TGF alfa y beta en la mediación del desarrollo de conductos y alvéolos. La TGF no solo promueve el crecimiento de los alvéolos que se desarrollan, sino que también puede mediar la apoptosis e involución para facilitar la diferenciación. Además de sus efectos sobre el crecimiento ductal, la TGF también puede inhibir la lactancia. Para el momento del nacimiento, el tejido mamario neonatal se ha hecho funcional y puede secretar el calostro para la primera semana de vida. Este fluido consiste en restos celulares y comúnmente es llamado leche de bruja. Esto puede alarmar a los padres, pero no es considerado un hallazgo patológico. El tejido mamario en la niñez crece al mismo ritmo que el cuerpo, pero deja de segregar siempre que los niveles de estrógeno permanezcan bajos. Los niveles anormalmente altos de estrógeno, sin embargo, pueden hacer que el tejido mamario inmaduro prolifere y madure, causando el desarrollo de ginecomastia o pubertad precoz.

\section{ANATOMÍA DE LA MAMA (Figura 3)}

Límites Los límites anatómicos de la mama adulta están entre el borde esternal y la línea media axilar y entre la segunda y la sexta costilla. Los límites quirúrgicos incluyen la clavícula a nivel superior, el pliegue inframamario a nivel inferior, el borde del dorsal ancho y el borde esternal medio en los laterales. La parte de la mama que se proyecta en la axila es llamada cola de Spence. Aunque el pezón se localice en la proyección del cuarto espacio intercostal, en mamas péndulas la posición (ubicación) del pezón puede variar extensamente

Mientras la mama tiene componentes significativos de tejido de la piel y subcutáneo, también está formada de tejido mamario específico: parénquima y estroma. El parénquima mamario está formado por lóbulos, que a su vez están constituidos por alvéolos. Entre 20 y 40 lobulillos constituyen un lóbulo, y los lóbulos pueden ser agrupados en segmentos. Estos se ubican radialmente alrededor del pezón y hay 15 a 20 por mama. Los conductos colectores drenan cada segmento, convergiendo en los senos galactóforos que a su vez drenan en conductos de galactóforos. El parénquima mamario, con la grasa circundante, recubre la fascia pectoral profunda y es envuelto por la fascia pectoral superficial. Las "bandas" de tejido fibroso, llamadas ligamentos de Cooper, atraviesan la fascia superficial y profunda pectoral, y actúan como sostén mamario.

La irrigación está dada por dos arterias, la mamaria interna y la torácica lateral, las cuales suministran la mayor cantidad de sangre al tejido mamario. La arteria torácica lateral provee al cuadrante superior externo el $30 \%$ del suministro de sangre de la mama. Las regiones centrales y medias de la mama reciben sangre de la arteria mamaria interna, proveyendo el $60 \%$ del suministro de sangre. La rama pectoral de la arteria toracoacromial, las ramas laterales de las arterias intercostales, la arteria subescapular y la arteria toracodorsal proveen menor suministro.

La inervación sensorial de la mama anterior lateral y anterior medial es dada por las raíces nerviosas de los nervios D4 y D5. La parte superior del pecho por el nervio supraclavicular. El pezón recibe inervación sensorial de D4.

El drenaje linfático está dado por múltiples grupos linfáticos que drenan la mama. El plexo subepitelial es superficial y es una parte de la red linfática subepitelial que drena la superficie del cuerpo entero. El plexo subareolar drena el pezón y la areola, y desde el plexo subareolar la linfa fluye en el plexo subcutáneo perilobular y profundo. Estos sistemas drenan hacia los ganglios axilares (97\%) y mamarios internos (3\%).

\section{POSICIONAMIENTO TOPOGRÁFICO} (Figura 4)

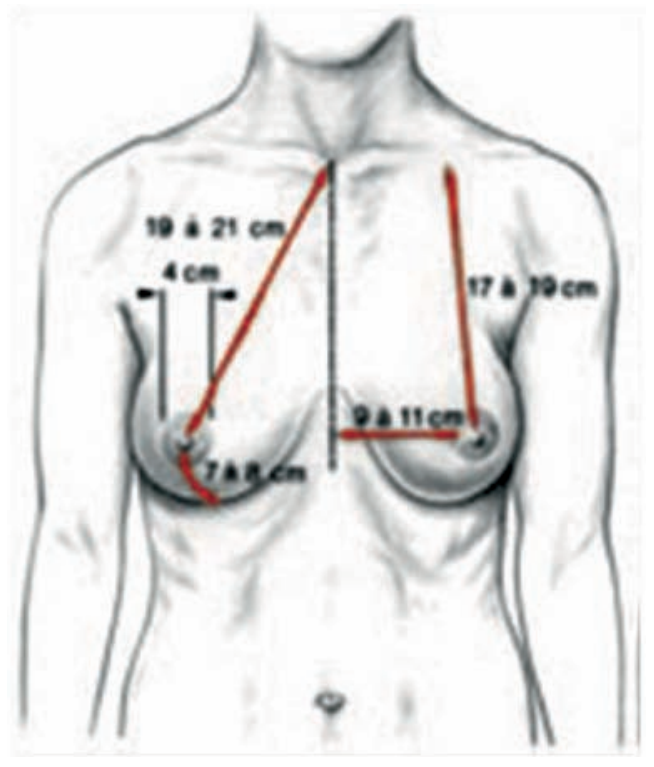

Figura 4. Posicionamiento. 


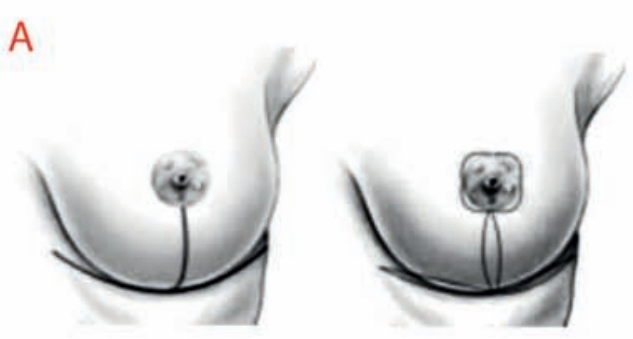

B
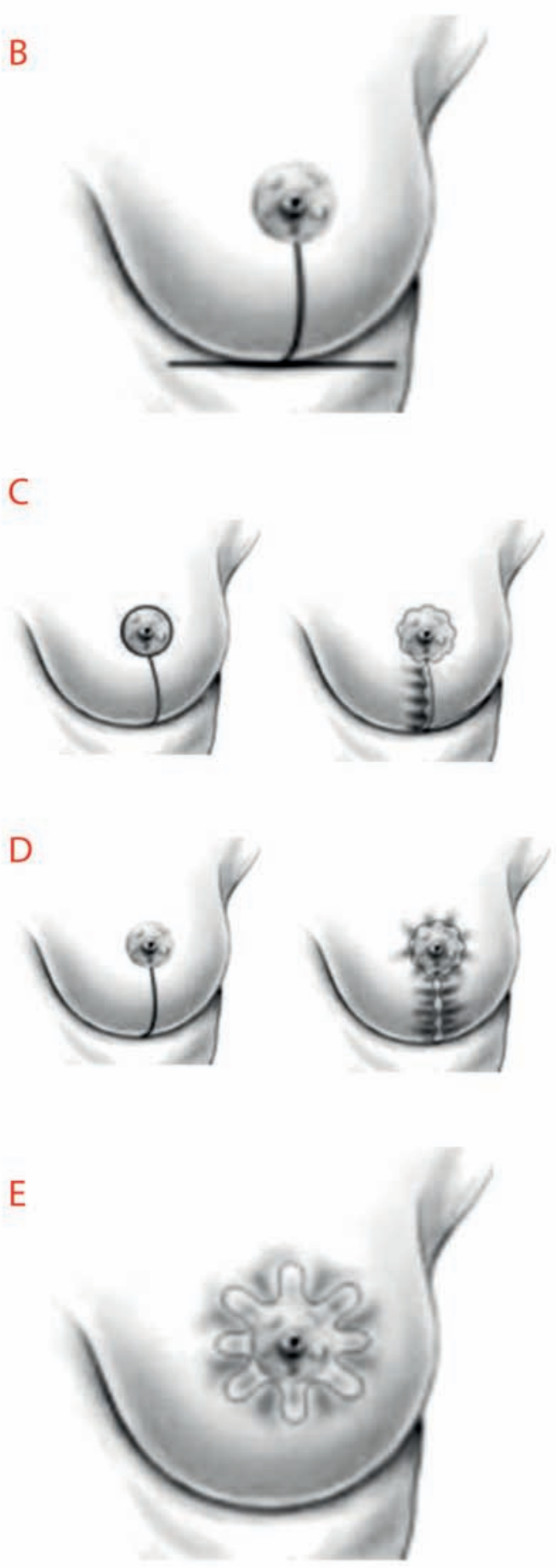

Figura 5. A, B, C, D, E. Técnicas según la cicatriz residual.

\section{PEXIA MAMARIA EN GLÁNDULA HIPERTRÓFICA}

Son numerosas las publicaciones de diferentes técnicas quirúrgicas para resolver la afección ptósica en glándulas hipertróficas, pudiendo dividirla en las técnicas que llevan el nombre de la cicatriz residual, las que aluden a la forma particular de la resección glandular y un tercer grupo que presenta como originalidad la forma de vascularización de los tejidos que quedan en su lugar después de la resección.

\section{TÉCNICAS SEGÚN LA CICATRIZ RESIDUAL.}

La cicatriz es una preocupación importante, tanto para la paciente como el cirujano. La cicatriz debe ser objeto de una elección basada en las indicaciones y motivo de información de la paciente, con una explicación muy precisa de su posición y tamaño, así como de sus posibles evoluciones, fisiológicas o patológicas. Todos los autores desaprueban las cicatrices en la parte superior supraareolar de la mama, visibles con los escotes, sometidas a tensiones excesivas y causa de evolución a la cicatriz hipertrófica o queloide. En todas las técnicas es necesaria la inevitable incisión periareolar, que requiere la transposición hacia arriba de la placa areolar, asumiendo que la hipertrofia se asocia siempre a cierto grado de ptosis. La absorción del excedente cutáneo, más o menos importante, explica la multiplicidad de las cicatrices.Las cicatrices «en ancla» consisten en una cicatriz horizontal larga, de la longitud del surco inframamario (Figura 5 A). Presenta el inconveniente de acercarse a la línea media, con riesgo de evolución hipertrófica. Por lo tanto, algunos autores han intentado acortar la cicatriz horizontal, como ocurre con las técnicas con cicatrices en «T invertida» o en «T invertida corta» ${ }^{4,5}$ (Figura 5 B). La supresión radical de la parte interna de la horizontal motiva las técnicas con cicatrices en L o en J (Figura $5 \mathrm{C}$ ). La rama externa y la parte vertical absorben las diferencias de longitud de los dos bordes. Muchos autores ${ }^{8,14,18,21}$ han descrito técnicas con una cicatriz vertical única (Figura 5 D), en las que se ha suprimido la parte horizontal. En estos casos, el problema es absorber el excedente cutáneo intentando eliminar el tejido adiposo subcutáneo y retrayendo los planos liberados. El fruncido sobre la línea vertical es máximo y se concentra sobre todo en la parte inferior de la cicatriz (bolsa), lo que ocasiona una maduración más larga. Son frecuentes las correcciones cicatriciales con la creación de un corte horizontal. Se deben elegir las indicaciones adecuadas ${ }^{16}$. La supresión de la línea horizontal, y también de la vertical, han motivado el perfeccionamiento de las técnicas periareolares (Figura $5 \mathbf{E})^{10}$, en las que todo el excedente cutáneo es absorbido en la cicatriz periareolar, expuesta de este modo a tensiones excéntricas y, por lo tanto, a una expansión de la placa areolar y de la cicatriz, sin contar el aplanamiento de la zona de concentración. 

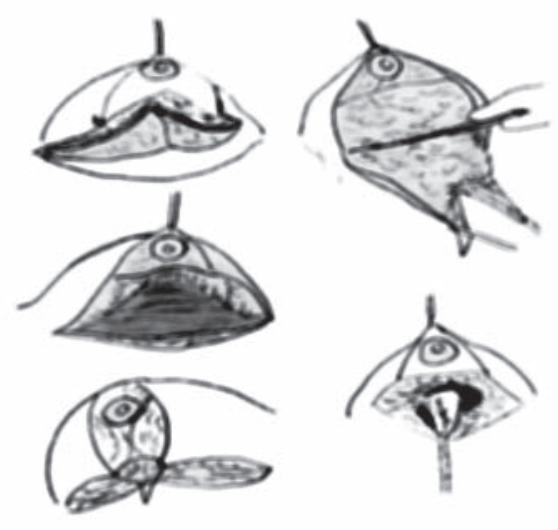

Figura 6. Técnica de Pitanguy.

\section{TÉCNICAS SEGÚN EL MODO DE RESECCIÓN GLANDULAR}

La ablación del excedente glandular debe permitir el respeto de la vascularización del tejido glandular y de la placa areolar restante y la realización de una masa hística residual bastante flexible para ser remodelada y suficientemente vascularizada para evitar las complicaciones necróticas. Desde la resección externa, propuesta originalmente por Biesenberger ${ }^{1}$, se han propuesto numerosas formas de vaciamiento o de resección en bloque: la resección en «quilla de barco invertida» de Pitanguy ${ }^{3}$ (cuya ventaja consiste en permitir la remodelación incluso en los casos de marcada distrofia fibroadiposa), resección en cuña, las resecciones en «reloj de arena» ${ }^{13}$, en «herradura» $»^{22}$, en «asa ${ }^{13}$, resección posterior con lámina dermoglandular ${ }^{6}$, entre otras.

\section{TÉCNICA DE PITANGUY3 (Figura 6)}

Marcaje: con la paciente en la mesa de quirófano con $45^{\circ}$ de inclinación y los brazos en $90^{\circ}$ con respecto la axila. Se marca el punto medio de la clavícula y se traza una línea perpendicular a dicho punto que llegue al surco submamario. A nivel de dicha línea en el surco submamario se presiona suavemente hacia arriba con el dedo índice de una mano y con el dedo índice de la otra mano se hace coincidir con el dedo índice de la otra mano dibujando en este punto marcamos el punto A. El cirujano marca dos puntos equidistantes en línea perpendicular a la areola C y B. Los puntos D, extremo medial del surco submamario, y E, extremo lateral del surco submamario, deben ser equidistantes. Puntos D y E se conectan así como B-E y C-D.

Técnica quirúrgica: el ayudante sujeta con presión la mama desde su base axilar y medial dejando tensa el área A-B-C y se realiza disección de la piel con el bisturí entre la epidermis y dermis desepitelizando dicha área de resección. El ayudante tracciona la mama desde el punto A de forma perpendicular a la parrilla costal; con un bisturí se incide desde el punto $\mathrm{C}$ al $\mathrm{D}$ así como de $\mathrm{B}$ a $\mathrm{E}$, se continúa la incisión de $\mathrm{D}$ a $\mathrm{E}$ continuándo-

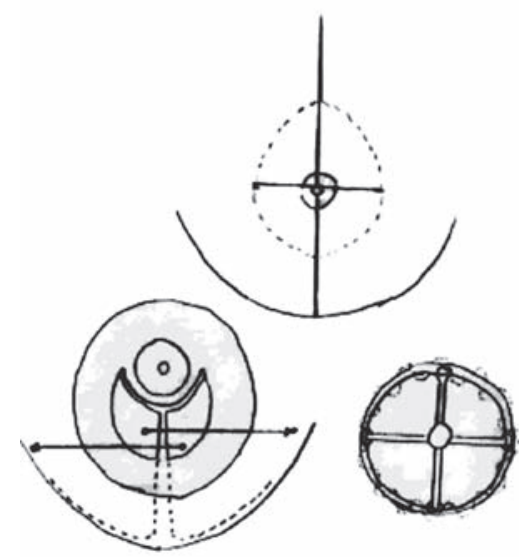

Figura 7. Técnica de Benelli.

se en profundidad con una resección en quilla hasta el pectoral mayor. Por último, se cierra por planos.

\section{Biesenberger ${ }^{1}$}

Realizaba una completa disección de la piel de la mama. Posteriormente realizaba una resección del parénquima mamario en forma de $S$ en el polo lateral: para reconstruir la forma cónica de la mama la porción remanente del polo inferior se rotaba hasta juntarlo con el borde remanente en el polo superior quedando de esta manera la areola en su nuevo emplazamiento. Después de obtener la forma deseada el exceso de piel era resecado quedando una cicatriz en T invertida.

\section{Round block (Benelli) ${ }^{10}$ (Figura 7)}

Técnica indicada en hipertrofias moderadas con ptosis grado I.

Marcaje: se marca una línea desde la mitad de la clavícula hasta el borde submamario, el punto donde se localizará el nuevo pezón es la proyección del surco submamario sobre dicha línea. Se marcan los siguientes puntos que determinarán el área a desepitelizar: punto A se localiza a $18 \mathrm{~cm}$ del reborde esternal; punto B o borde medial se marca a $8 \mathrm{~cm}$ del esternón; el borde lateral se determina por el punto $C$, que se marca a 10 $\mathrm{cm}$ de la línea axilar el borde inferior o punto $D$, a $6 \mathrm{~cm}$ del surco submamario. Se unen los puntos y determinamos el área a desepitelizar.

Técnica quirúrgica: se realiza una incisión en el borde externo del área desepitelizada Se realiza una resección en cuña bajo la areola, los bordes de resección del tejido mamario se suturan al periostio de la costilla subyacente, el borde lateral al periostio esternal y el medial al periostio de la costilla en su borde lateral realizando un crisscrossing. Cierre de la incisión formando una cicatriz periareolar.

\section{TÉCNICA SEGÚN EL PEDÍCULO BASCULAR DEL TEJIDO REMANENTE}

En ellas la unidad piel-glándula se va a mantener, el 


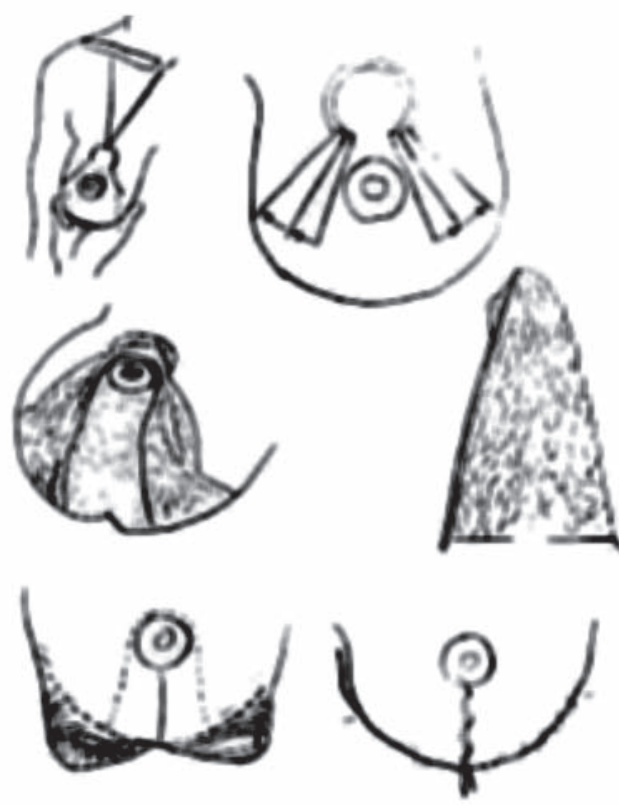

Figura 8. Técnica del pedículo inferior

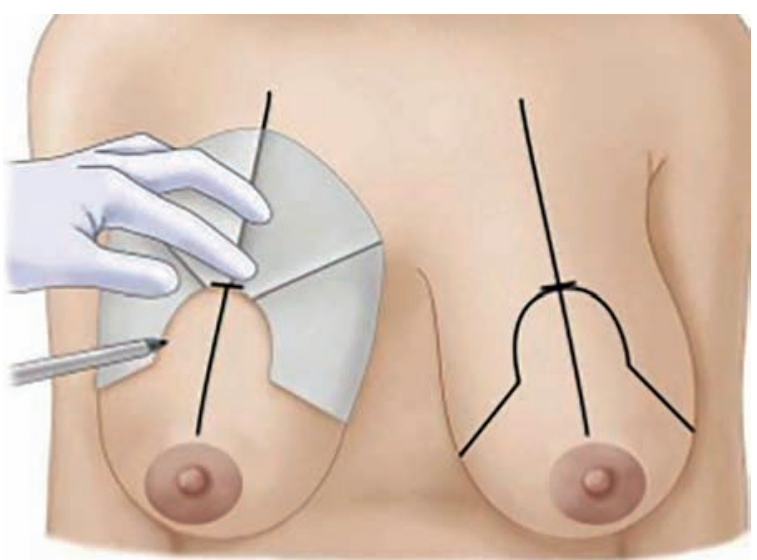

Figura 9. Marcación según el patrón de Wise.

plano de despegamiento se va a realizar entre el plano muscular y el glandular. Distinguiremos: técnicas de pedículo inferior, técnicas bipediculadas y técnicas de pedículo superior $22,7,11,12,15,9$.

\section{Pedículo inferior (Figura 8)}

Se utilizan en las grandes hipertrofias mamarias, por la seguridad que supone la preservación del complejo areola-pezón.

Marcaje: tras el marcaje según patrón de Wise (Figura 9), se dibuja el pedículo con una base de $7-10 \mathrm{~cm}$. Desde el surco submamario asciende hasta la areola circunvalándola con un margen de $1 \mathrm{~cm}$.

Técnica quirúrgica: se desepiteliza el pedículo iniciándose la resección de la glándula mamaria en los márgenes del área desepidermizada y el marcaje previamente realizado.

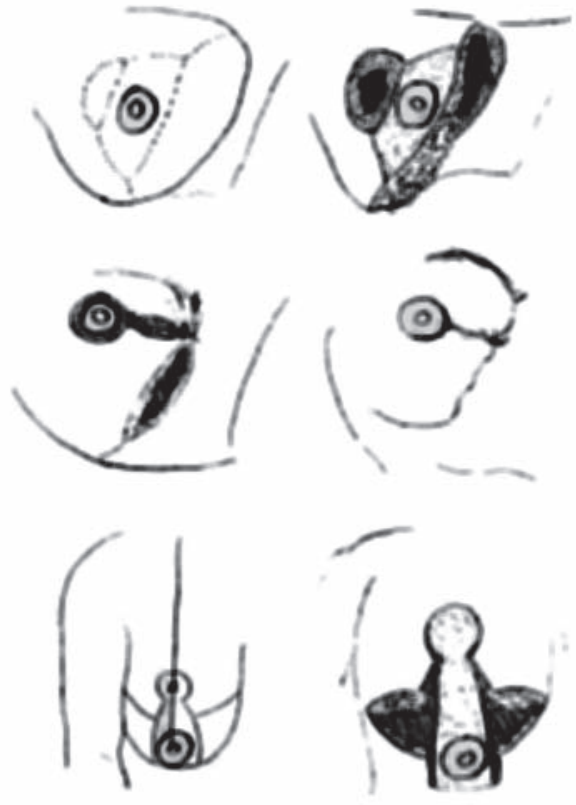

Figura 10. Técnica de Strombeck

El pedículo se adelgaza en el ámbito de la areola con 2-3 cm de espesor y de ahí irá creciendo en espesor hasta alcanzar en la base $8-10 \mathrm{~cm}$. Se asciende el pedículo hasta su nueva situación y los colgajos verticales se suturan al punto medio del surco submamario. Se cierra por planos la piel y la areola.

\section{Bipediculado / pedículo interno Bipediculados}

Técnica de Strömbeck ${ }^{2}$ (Figura 10): Strombeck revolucionó las técnicas de mamoplastia de reducción al proponer una técnica de transferencia de la areola sobre un colgajo dermoglandular horizontal, que después se transformó en un colgajo de pedículo interno. Se realiza el marcaje clásico con el patrón de Wise.

Técnica quirúrgica: la operación se inicia con una incisión periareolar de unos $4-5 \mathrm{~cm}$ de diámetro, los pedículos se diseñan como un puente horizontal entre el borde lateral y medial del colgajo cutáneo. Este puente se desepiteliza, realizando la resección glandular en el polo inferior y superior incidiendo hasta el plano del pectoral. La trasposición del CAP se realiza con un desplazamiento horizontal hacia arriba del complejo areola-pezón y se lo coloca en el área creada previamente para su implantación.

Ventajas: está indicada para grandes y moderadas hipertrofias.

Inconvenientes: es difícil de aplicar en mamas con un 


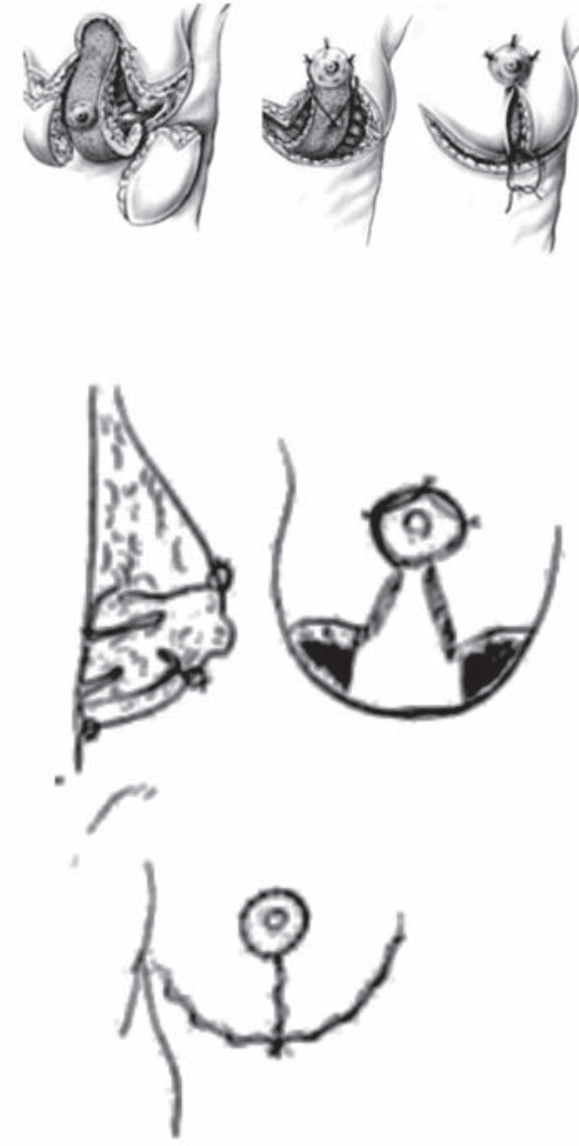

Figura 11. Técnica de Mc Kissock.

gran componente graso. El porcentaje de insensibilidad areolar es grande.

\section{Técnica de Mc Kissock (Figura 11)}

La técnica se puede aplicar en todas las mamas; máxima vascularización del pedículo.

Marcaje: básicamente el marcaje es idéntico al anterior una vez hechas la marcas de la mamoplastia convencional se trazan las marcas del pedículo vertical la base del pedículo inferior tiene que tener una longitud de $5 \mathrm{~cm}$ de ancho y dependiendo del tipo de mama se dibujara el pedículo superior, dicho dibujo dependerá de la distancia que tengamos que subir el CAP, de tal forma que en mamas moderadas con ptosis pequeñas no será necesario separar el pedículo del ojo de llave de la nueva implantación del CAP en mamas grandes y ptósicas se realizará un puente vertical el cual medirá unos $5 \mathrm{~cm}$ de ancho.

Técnica quirúrgica: se desepiteliza el pedículo, se tracciona con un kocher la mama desde la parte superior del pedículo superior y se inicia la resección empezando por el

tejido mamario por debajo del CAP dejando un panículo delgado en anchura en la totalidad del pedículo vertical. En el resto de la mama se realiza una resección

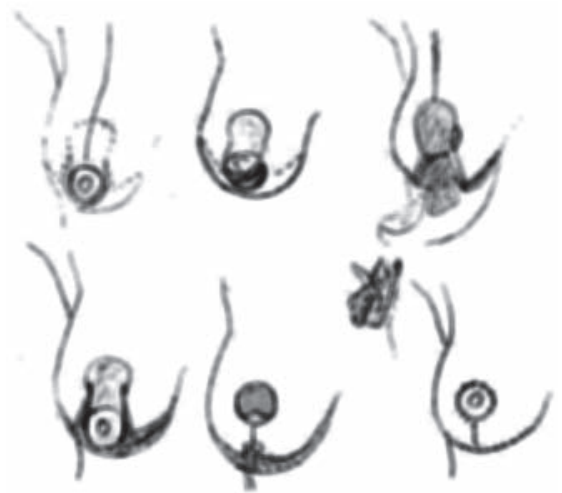

Figura 12. Técnica del pedículo superior.

considerando la cantidad a resecar y la forma definitiva de la mama. La trasposición

del complejo areola-pezón se facilita con dos incisiones paralelas a ambos lados del ojo de cerradura que será la nueva posición del CAP. Se suturan los colgajos cutáneos laterales (de $5 \mathrm{~cm}$ máx. cada uno) al surco submamario y se introduce el complejo areola-pezón en su nueva posición.

Ventajas: seguridad vascular de la areola. Facilidad en su ejecución.

Inconvenientes: las grandes cicatrices submamarias. $\mathrm{Di}$ ficultad para plegar el colgajo adecuadamente. Riesgo importante de desarrollo del segmento inferior por la basculación hacia la parte superior de la areola.

\section{Técnica del pedículo superior (Figura 12)}

Se realiza el marcaje según técnica tradicional dibujando un pedículo de base en el ojo de cerradura del dibujo y prolongándose hasta $1 \mathrm{~cm}$ de margen del CAP. Se preservará con el complejo areola-pezón con un espesor de $1,5 \mathrm{~cm}$ para posteriormente resecar todo el tejido glandular subyacente que queramos resecar, tanto del polo inferior como de los cuadrantes inferiores, reconstruyendo la forma de la mama con puntos entre los pilares lateral y medial. El colgajo de $1,5 \mathrm{~cm}$ de espesor se sitúa en su nueva posición realizando dos incisiones laterales de $1 \mathrm{~cm}$ a cada lado para permitir su ascenso en su nueva posición. Una vez ascendido, siendo ésta una de las maniobras más cuidadosas a realizar, montamos la areola en su nueva ubicación realizando sutura primero con puntos no reabsorbibles, en los cuatro puntos cardinales a la piel. Se termina suturando por planos.

Ventajas: presenta mayor relleno del polo superior y proyección de la mama.

Inconvenientes: presenta mayor riesgo de necrosis del CAP.

PREDILECCIÓN DE TÉCNICA SEGÚN ASOCIACIÓN DE HIPERTROFIA Y PTOSIS MANIFIESTA ${ }^{21}$

En 1998 se realizó un sondeo entre 200 cirujanos plás- 
ticos norteamericanos. En cuanto a la técnicas más utilizadas manifestaron que:

- Un $74 \%$ realizaba pedículo inferior con incisión en T invertida.

- Un $22 \%$ realizaba pedículo superior, de los cuales un $9 \%$ realizaban incisión en $\mathrm{T}$ y un $12 \%$ incisión vertical.

\section{Técnica utilizada para pequeñas hipertrofias:}

- Pedículo inferior con T invertida $43 \%$.

- Pedículo superior $47 \%$ de los que $32 \%$ son con cicatriz vertical.

- Periareolar 10\%.

\section{Hipertrofias moderada y ptosis grado II}

- Pedículo inferior con incisión en T 84\%.

- Pedículo superior 16\% de las que 9\% son con incisión vertical.

\section{Moderada macrosmática con ptosis grado III}

- Pedículo inferior con incisión en T 80\%.

- Pedículo superior $12 \%$ de las que el $8 \%$ son con incisión vertical.

- Injerto libre de CAP 8\%.

\section{Hipertrofias grandes con ptosis II III}

- Pedículo inferior con T invertida 49\%.

- Pedículo superior con T invertida 8\%.

- Injerto libre de CAP 43.

\section{PEXIA MAMARIA EN GLÁNDULA HIPOTRÓFICA / ATRÓFICAS}

\section{PTOSIS MAMARIA POR ATROFIA GLANDULAR}

En las ptosis mamarias como única afección, la reducción de la envoltura cutánea se realiza según el esquema preestablecido con las mismas referencias: línea interna, punto clave, línea externa, que frecuentemente es tangente al borde externo de la areola o está en su proximidad (1 a $2 \mathrm{~cm}$ ). La horizontal externa es corta y se traza en el surco y no por encima de él. En los casos en los que la base de la mama es estrecha puede ser suficiente una vertical. La glándula mamaria es luxada de la misma manera que en las hipertrofias, para liberarla de su hemisferio inferior. A continuación, simplemente se envuelve en su envoltura cutánea, sin colgajo ni sutura glandular. La tensión debe ser firme a nivel de la sutura del segmento III, que será plano para prevenir la ptosis secundaria.

Técnica quirúrgica: El tratamiento quirúrgico para la reparación de las ptosis mamarias va a consistir en extirpación de la piel, colocación de la areola en su posición inicial, plicatura y fijación de la mama para evitar una caída de la misma.

Vamos a distinguir dentro del tratamiento de las ptosis mamarias, aquellas que se van a poder resolver solo con la remodelación de la propia mama, extirpación de la piel sobrante, y colocación de su areola, de aquellas que citaremos en segundo lugar y que conllevan la colocación de un implante mamario que va a permitir el relleno del polo superior de la mama.

\section{MASTOPEXIA SIN IMPLANTE MAMARIO. ${ }^{13,23,24}$}

Basándonos en la clasificación de Regnault, vamos a establecer según los tres grados de ptosis, los tratamientos a realizar:

GRADO I. Puede ser suficiente con la desepitelización de la areola superior, y su colocación en un plano superior. Técnica del doughnut, mastopexia periareolar (Hinderer, 1969-1972).

GRADO II. En estos casos será necesario subir la areola y la extirpación de la piel sobrante. Para ello se han descrito múltiples técnicas: Arie (1962). Hinderer. (1976), con la paciente en bipedestación, marcaje de una línea central que va desde el tercio medio de la clavícula, pasando por la areola hasta el pliegue inframamario. Marcaje del surco inframamario. Se ubica la nueva areola, y marcaje de la piel con el patrón de Wise. Resección de piel y realización de un colgajo dermoglandular de pedículo interno y superior que se ancla tras el despegamiento de la glándula en la fascia del músculo pectoral mayor. Es importante realizar un gran despegamiento que es lo que va a permitir el remodelado de la glándula mamaria. Sutura por planos. GRADO III, la ptosis en este caso es mayor por lo que las técnicas utilizadas serán las mismas que las anteriores, pero con la extirpación de mayor cantidad de piel. Las técnicas que permiten la corrección de las grandes ptosis mamarias tienen que favorecer que la mama permanezca en posición a lo largo del tiempo, por lo que vamos a citar la técnica de Rodrigo Cucalón. Consiste en la remodelación de lamama en base a los extremos laterales del remanente del polo inferior de la misma. Pasamos a describir la técnica: desepidermización hasta la porción inferior de las ramas verticales del patrón de Wise; luego sección de la dermis entre las ramas verticales y elevación de un colgajo dermoglandular del pedículo superior que incluye el complejo areola-pezón. Se completa el despegamiento de los colgajos cutáneos, de la glándula, llegando hasta la clavícula. Los extremos laterales de la porción inferior de la glándula se suturan sobre sí mismos, y una vez dada la forma de la "pelota" se ancla la glándula al músculo pectoral mayor por medio de una semicorona de puntos que se interrumpe en ambos extremos al llegar al surco submamario (que permanece en su lugar de origen). La glándula queda así fija y tersa.

\section{MASTOPEXIA Y PRÓTESIS 26,27}

Pasamos a describir la técnica utilizada en los casos en que se requiera para incrementar el volumen mamario, la utilización de un implante mamario. Se utilizará la posición retropectoral del implante y las prótesis podrán ser de las distintas formas y rellenas de los distintos materiales de los que disponemos en el mercado. Se 


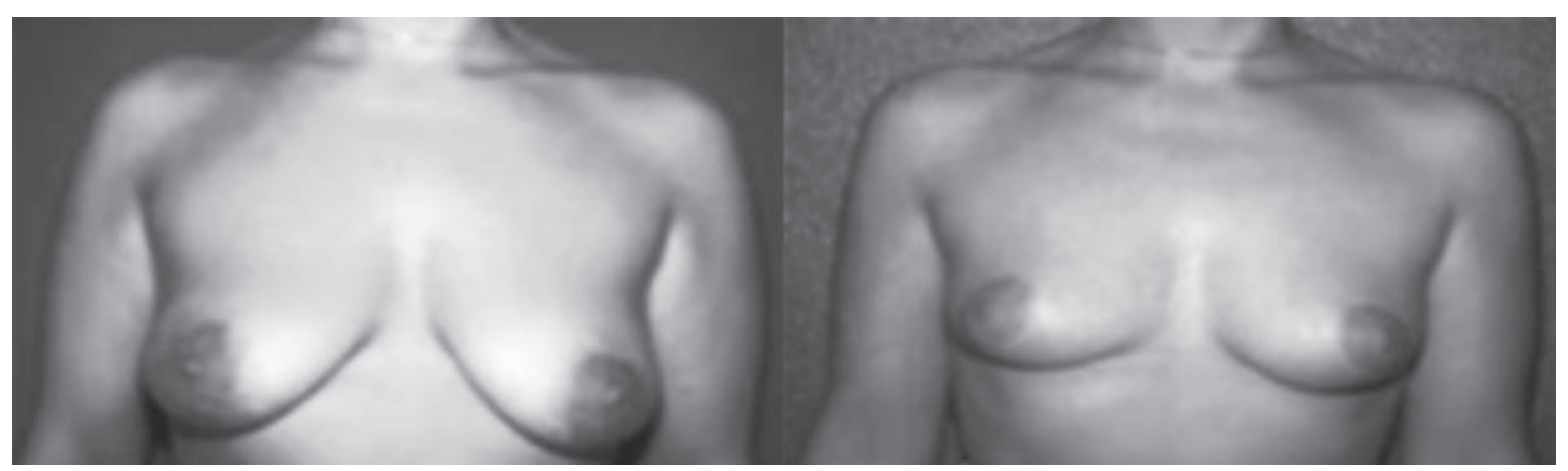

Figura 13. Resultados quirúrgicos de una marcación adecuada

marcará previamente a la paciente en bipedestación la altura de la futura ubicación de la areola, Realizaremos el marcaje con los patrones utilizados. En quirófano y con la paciente en decúbito supino, se realiza pequeña incisión oblicua en el polo inferior de la mama por donde se colocará la prótesis. Despegamiento y disección hasta llegar al borde del músculo pectoral mayor. Es conveniente en los casos que tenemos poco componente glandular colocar los implantes por debajo del músculo, ya que ello nos permitirá que estén más recubiertos. Despegamiento en el plano retropectoral y colocación de la prótesis. Ahora es necesario saber la cantidad de piel sobrante e iniciar la mastopexia. Marcaje de la mama. Desepidermización y elevación del complejo areola-pezón. Marcaje de un colgajo de pedículo interno, despegamiento del plano subglandular del plano subpectoral. Introducción del colgajo que rellena la parte posterior del complejo areola y pezón y lo impulsa hacia adelante dándole una forma cónica a la mama. Sutura por planos.

\section{COMPLICACIONES 25}

Además de todas las complicaciones de la cirugía en sí, como pueden ser hematomas, infección, seromas, hay que decir que las principales y específicas complicaciones de la cirugía en las ptosis mamarias serían:

Mama ptósica. Tras la realización de la técnica correc- tora, es posible que si no se ha realizado bien y la glándula no ha sido bien sustentada, vuelva a caer con el tiempo.

En los casos en que se ha colocado prótesis mamaria, tenemos que prever la caída natural que conlleva el descenso de la inflamación de la mama tras la colocación de la prótesis, que puede ser la causa de que marquemos el complejo areola-pezón en un sitio erróneo. En estos casos habrá que sobreañadir cualquiera de los efectos y complicaciones que puedan conllevar la colocación de las prótesis mamarias. (desplazamientos, roturas, etc.).

\section{CONCLUSIÓN}

En materia de cirugía para la corrección de la ptosis, ya sea por atrofia o hipertrofia glandular, la elección de la técnica depende de las condiciones locales, a la vez cutáneas (elasticidad), glandulares (plasticidad) y morfológicas (armonía).

Según lo desarrollado conforme existen numerosas técnicas para la resolución de una misma patología hace al entendimiento de que cada paciente constituirá un ente individual al cual se debe someter al análisis exhaustivo para determinar cuál técnica quirúrgica será la de mayor provecho para la resolución de su afección, logrando el mejor resultado estético posible.

\section{BIBLIOGRAFÍA}

1. Biesenberger H. Eine neue Methode der Mammaplastik. Zentralbl Chir 1930.

2. Strombeck JO. Mammaplasty: report of a new technique based on the two pedicle procedure. 1960.

3. Pitanguy I. Une nouvelle technique de plastie mammaire: etude de 245 cas consecutifs et presentation d'une technique personelle. Ann Chir Plast 1962.

4. Llalardrie JP, Jouglard JP. Chirurgie plastique du sein, rapport de la Societe Francaise 1973

5. Lalardrie JP, Mitz V. Plastie mammaire de reduction par la technique de la voûte dermique. 1974.

6. Lalandrie JP. Mitz V. Plastie mammaire de reduction par la technique de la voute dermique 1974
7. Eiseman G. Augmentation mammaplasty by transaxillary approach. Plastic. Reconstr. Surg. 54: 229,1974.

8. lassus C. Breast reduction. Evolution of a technique. A simple vertical scar. 1987.

9. McCarthy JG. Plastic Surgery vol. 6. Philadelphia: Ed. W.B. Saunders; 1990.

10. Benelli L. Periareolar mammaplasty: Round bock technic. Aesthe tic Plast Surg 1990;14:99.

11. Georgiade. Aesthetic Surgery of the Breast. Philadelphia: Ed. W.B. Saunders; 1990.

12. Grabb and Smith's plastic surgery. Boston/Toronto/London: Ed. Little Brown; 1992. 
13. Strombeck JO, Rosato FE. Surgery of the Breast. New York: Ed. Thieme; 1992.

14. Lejour M. Vertical mammaplasty and liposuction of the breast. Plast Rec Surg 1994;94:100.

15. Bohmert H. Plastic and reconstructive surgery of the breast. Christian Gabka. New York: Ed Thieme; 1997.

16. Lejour M. Vertical mammaplasty: early complications after 250 personal consecutive cases. Plast Reconstr Surg 1999 Sep;104(3):76470.

17. Cirugía plástica reparadora y estética. EMC Paris, 1999.

18. lassus C. Update on vertical mammaplasty. Plast Reconstr Surg 1999.

19. Tabetts JB, Tabbbets TB. The breast. Dallas: Ed. Cosmetxpertise; 1999.
20. Giovanoli P, Meuli-Simmen C, Meyer VE, Frey M. Which technique for which breast? Br J Plast Surg 1999 Jan;52(1):52-9.

21. Cunningham B, Lokeh A, Gutowsky K. Saline-filled breast implant safety and efficacy: a multicenter retrospective review. Plast Reconstr Surg 105: 2143, 2000.

22. Mizgala $C L$, MacKenzie KM. Breast reduction outcome study. Ann Plast Surg 2000 Feb;44(2):125-33.

23. Rohrich RJ. Approves saline-filled breast implants: What does this linean for our patients. Plastic Reconstr Surg 2000;106:9030.

24. U.S. Food and Drug Administration. Breast Implant Complications 2000.

25. U.S. Food and Drug Administration. Breast Implants 2000.

26. www.cirugias de la mama.com/elevación/mamaria. 